\title{
Elektronische Tagebücher optimieren die Kognitive Verhaltenstherapie bei Krankheitsängsten: Eine randomisiert-kontrollierte Studie
}

\author{
Josef Bailer ${ }^{1}$, Tobias Kerstner ${ }^{1}$, Daniela Mier ${ }^{1}$, Fred Rist ${ }^{2}$, Carsten Diener ${ }^{3}$, \\ Gaby Bleichhardt ${ }^{4}$ und Michael Witthöft ${ }^{5}$
}

\begin{abstract}
${ }^{1}$ Abteilung Klinische Psychologie, Zentralinstitut für Seelische Gesundheit, Mannheim
${ }^{2}$ Psychologisches Institut I, Wilhelms-Universität, Münster

${ }^{3}$ Fakultät für Angewandte Psychologie, SRH Hochschule Heidelberg

${ }^{4}$ Arbeitsgruppe Klinische Psychologie und Psychotherapie, Philipps-Universität, Marburg ${ }^{5}$ Abteilung Klinische Psychologie, Psychotherapie und Experimentelle Psychopathologie,
\end{abstract} Johannes Gutenberg-Universität, Mainz

\begin{abstract}
Zusammenfassung. Zentrale Komponenten in kognitiv-behavioralen Erklärungsmodellen der Hypochondrie und den daraus abgeleiteten Behandlungen sind eine selektive Aufmerksamkeitslenkung auf körperliche Empfindungen und ein katastrophisierender Symptominterpretationsbias. Wir untersuchten, ob sich die Effektivität einer störungsspezifischen Kognitiven Verhaltenstherapie (KVT) für Patienten mit Hypochondrie durch ein zusätzliches zweiwöchiges elektronisches Tagebuchtraining (TBT), das auf eine Korrektur der Symptominterpretationen abzielt, verbessern lässt. Zu diesem Zweck wurden 35 Patienten mit Hypochondrie randomisiert zwei Interventionsformen zugewiesen: Gruppe $1(N=16)$ erhielt vor der KVT (16 Einzelsitzungen) ein zweiwöchiges TBT unter therapeutischer Anleitung (4 Sitzungen). Gruppe $2(N=19)$ erhielt vor der KVT vier Therapiegespräche ohne TBT. Das Ausmaß der Krankheitsangst wurde mit dem Short Health Anxiety Inventory erfasst. Beide Gruppen zeigten nach der Therapie eine signifikante Reduktion der Krankheitsangst. In der Gruppe mit TBT verbesserte sich die Krankheitsangst jedoch schneller $(d=.75)$ und stärker $(d=.76)$ als in der Kontrollgruppe. Zudem zeigte die Gruppe mit TBT eine stärkere Habituation an bedrohliche Symptomstimuli als die Kontrollgruppe. Weitere Studien an größeren Stichproben sowie die Untersuchung von Langzeiteffekten sind notwendig, um die Wirksamkeit des vorgestellten TBT zu belegen.
\end{abstract}

Schlüsselwörter: Hypochondrie, elektronische Tagebücher, Reattributionstraining, Kognitive Verhaltenstherapie

Electronic diaries enhance the effect of Cognitive Behavioral Therapy for health anxiety: A randomized controlled study

\begin{abstract}
Selective attention allocation towards bodily sensations and a catastrophic symptom attribution bias are central components of cognitive-behavioral models of hypochondriasis. We investigated whether the effectiveness of a specific cognitive-behavioral psychotherapy (CBT) for patients with hypochondriasis can be enhanced by adding a specific 2-weeks electronic-diary based training (EDT) in order to correct the symptom attribution bias. Thirty-five patients with hypochondriasis were randomly assigned to two intervention groups: Group $1(N=16)$ received the EDT that was explained and supervised in four therapeutic sessions. Group $2(N=19)$ received four therapeutic sessions without the EDT. After these four sessions, both groups received CBT for health anxiety. The level of health anxiety was assessed with the Short Health Anxiety Inventory. After therapy, in both groups, a significant reduction of health anxiety was observed. The EDT group showed a faster $(d=.75)$ and stronger $(d=.76)$ reduction of health anxiety compared to the control group. Additionally, the EDT group showed a stronger habituation to threatening symptom words compared to the control group. Future studies with larger sample sizes and the investigation of long-term effects are necessary to confirm the effectiveness of the proposed EDT.
\end{abstract}

Keywords: hypochondriasis, health anxiety, electronic assistants, symptom reattribution training, cognitive behavioural therapy 
Krankheitsängste und Krankheitsüberzeugungen, die auf einer Fehlinterpretation von körperlichen Beschwerden beruhen und trotz angemessener medizinischer Abklärung und ärztlicher Rückversicherung fortbestehen oder schon nach kurzer Zeit erneut auftreten, sind das zentrale Merkmal hypochondrischer Störungen (APA, 2000; Fink et al., 2004; WHO, 1993). Diese klinisch relevanten Störungen sind insbesondere bei Patienten in Haus- und Facharztpraxen häufig zu beobachten (Creed \& Barsky, 2004; Fink et al., 2004; Tyrer et al., 2014). Die Störungen verlaufen oft chronisch (olde Hartman et al., 2009) und die Therapieprognose galt lange Zeit als ungünstig (Bouman, 2014; Neng \& Weck, 2014). Im aktuell gültigen DSM-5 wurde die Diagnose Hypochondrie aufgegeben. Für Patienten mit pathologischen Krankheitsängsten ist nun in Abhängigkeit davon ob zusätzlich stark belastende Körpersymptome vorliegen oder nicht entweder die Diagnose der «somatic symptom disorder» oder die Diagnose der «illness anxiety disorder» vorgesehen (APA, 2013).

Durch die Entwicklung kognitiv-behavioraler Störungsmodelle (z.B. Warwick \& Salkovskis 1990; Williams, 2004) und daraus abgeleiteter Behandlungskonzepte konnten in den letzten Jahrzehnten in der Therapie der Hypochondrie beachtliche Fortschritte erzielt werden. In einem Cochrane-Review (Thomson \& Page, 2009) zu sechs randomisiert-kontrollierten Studien zeigten sich die kognitiven und verhaltenstherapeutischen Interventionen gegenüber den Kontrollbedingungen (Warteliste, Routineversorgung, Placebo) als deutlich überlegen. Die mittlere Effektstärke (Hedges' $g$ ) in der Reduktion hypochondrischer Symptomatik lag mit 0.86 im Bereich großer Effekte. Ähnliche Effektstärken (Hedges' g: 0.68-0.73) wurden für die Kognitive Verhaltenstherapie (KVT) bei Angststörungen und Depressionen ermittelt (Haby, Donnelly, Corry \& Voss, 2006; Hofmann \& Smits, 2008).

Dennoch stellt sich die Frage, wie die Wirkung von KVT bei hypochondrischen Störungen noch verbessert werden kann. Wir denken hier vor allem an eine Erweiterung der KVT um emotionsfokussierte Verfahren, die der «dritten Welle» der Verhaltenstherapie zuzurechnen sind, wie die Achtsamkeitstherapie (McManus, Surawy, Muse, Vazquez-Montes \& Williams, 2012; Williams, McManus, Muse \& Williams, 2011) und die Acceptance und Commitment Therapie (Eilenberg, Kronstand, Fink \& Frostholm, 2013) sowie an den Einsatz von elektronischen Assistenten (Handheld PCs; Wilhelm \& Perrez, 2008) und internetbasierter KVT (Hedman et al., 2011, 2014).

Bislang ist nur wenig darüber bekannt, welche Faktoren darüber entscheiden, ob ein Therapieangebot von Betroffenen in Anspruch genommen wird oder nicht. Aus zwei Therapiestudien mit sehr großen Fallzahlen ist jedoch bekannt, dass die Inanspruchnahmerate von KVT bei krankheitsängstlichen Patienten eher gering ist. In einer Studie von Barsky und Ahern (2004) nahmen nur 30\% der geeigneten Teilnehmer ein sechs KVT-Sitzungen um- fassendes Behandlungsangebot an. Die Nichtteilnehmer waren im Vergleich zu den Teilnehmern signifikant älter, weniger gebildet und häufiger männlich. Die KVT erwies sich im Vergleich zur medizinischen Routineversorgung als signifikant wirksamer hinsichtlich der Reduktion von Krankheitsangst und Krankheitsüberzeugungen. Diese Unterschiede waren auch in der 1-Jahres-Katamnese noch nachweisbar.

Tyrer und Kollegen (2014) berichten für ihre multizentrische Therapiestudie zur Behandlung von Krankheitsangst ebenfalls eine sehr hohe Ablehnungsrate: $68 \%$ der als hochkrankheitsängstlich eingestuften Patienten lehnten eine weitergehende Studienteilnahme ab. Die Autoren machten leider keine Angaben zu Merkmalen, die zwischen Teilnehmern und Nichtteilnehmern trennten. Die im Mittel ebenfalls nur sechs Sitzungen umfassende KVT erwies sich auch in dieser Studie gegenüber der Routineversorgung als überlegen. Die Vorteile zugunsten der KVT waren auch 2 Jahre nach Therapieende noch nachweisbar.

Die vielen «Nichtteilnehmer» schränken die Generalisierbarkeit der Ergebnisse solcher Studien allerdings ein und machen zugleich auf ein großes Versorgungsproblem aufmerksam, denn möglicherweise kann Personen, die für eine face-to-face-Therapie nicht zu gewinnen sind, durch andere Angebotsformen wie z.B. einer internetbasierten KVT geholfen werden (vgl. Hedman et al., 2011, 2013, 2014).

Ferner mangelt es an Studien, die sich mit der Überprüfung von Wirkmechanismen der KVT bei Hypochondrie beschäftigen. In kognitiv-behavioralen Modellen sind vier ineinandergreifende Mechanismen von zentraler Bedeutung für die Aufrechterhaltung von pathologischer Krankheitsangst: 1. Eine selektive Aufmerksamkeitsausrichtung auf körperliche Prozesse und Beschwerden. 2. Eine Fehlinterpretation objektiv harmloser körperlicher Veränderungen und Beschwerden als Anzeichen einer gravierenden körperlichen Erkrankung. 3. Sicherheitssuchendes Verhalten (z.B. Rückversicherung bei Ärzten oder in den Medien, Kontrollieren des eigenen Körpers) und 4. Vermeidungsverhalten (z.B. Vermeidung von Kliniken und anderen krankheitsassoziierten Reizen). Die ersten beiden Faktoren verursachen Angst, Unsicherheit und körperliche Erregung. Die letztgenannten Faktoren führen kurzfristig zur Angst- und Spannungsreduktion, langfristig jedoch zur Aufrechterhaltung von chronischen Krankheitsängsten und Krankheitsüberzeugungen.

Moderne Kognitive Verhaltenstherapien (z. B. Bleichhardt \& Weck, 2010) zielen dementsprechend darauf ab, diesen dysfunktionalen Kreislauf durch den Einsatz multimodaler Methoden zu durchbrechen. Diese umfassen Interventionen zur Aufmerksamkeitsumlenkung, kognitive Umstrukturierung fehlerhafter Symptominterpretationen und Grundannahmen, Expositionsübungen und Reduktion von Sicherheits- und Vermeidungsverhalten.

Neben der Wirksamkeitsüberprüfung solch komplexer Therapieprogramme sollte sich die zukünftige Forschung 
verstärkt auf die Untersuchung der Wirkfaktoren einzelner Interventionen richten. Bislang liegen hierzu jedoch nur wenige gut kontrollierte Studien vor. Die positive Wirkung von aufmerksamkeitsbezogenen Interventionen konnte jüngst in einer randomisiert-kontrollierten Studie an 36 Patienten mit Hypochondrie demonstriert werden (Weck, Neng \& Stangier, 2013). Die eine Hälfte der Patienten erhielt ein kurzes Aufmerksamkeitstraining, das drei wöchentliche 50-minütige Sitzungen umfasste, die andere Hälfte eine Intervention, die auf die Reduktion von Sicherheitsverhalten abzielte. In beiden Behandlungsbedingungen konnte eine signifikante Reduktion der Krankheitsängste nachgewiesen werden, aber die körperbezogene Aufmerksamkeit reduzierte sich nur in der Gruppe mit Aufmerksamkeitstraining $(d=.36)$. Eine weitere experimentelle Studie konnte zeigen, dass Sicherheitsverhalten zur Steigerung von Krankheitsängsten und krankheitsbezogenen Gedanken führt (Olatunji, Etzel, Tomarken, Ciesielski \& Deacon, 2011).

Vergleichbare Studien zur Veränderung des somatischen Interpretationsbias von hypochondrischen Patienten durch ein Attributionsmodifikationstraining sind uns nicht bekannt, obwohl ein ausgeprägter somatischer Symptomattributionsstil (Bailer et al., 2013a; Neng \& Weck, 2013) und katastrophisierende Symptominterpretationen (Rief, Hiller \& Margraf, 1998; Weck, Neng, Richtberg \& Stangier, 2012a; Weck, Neng, Richtberg \& Stangier, 2012b) charakteristisch für das Störungsbild sind.

Die vorliegende Studie ist Teil einer umfangreicheren Mehrebenen-Untersuchung zu attentionalen, affektiven, kognitiven und behavioralen Aspekten von Krankheitsangststörungen. In diesem Kontext beschäftigte uns auch die Frage, ob sich Krankheitsängste und emotionale Bewertungen von gesundheitsbedrohlichen Stimuli durch ein zweiwöchiges elektronisches Tagebuchtraining (TBT), das auf eine Korrektur der Symptominterpretationen abzielt, günstig beeinflussen lassen. Zu diesem Zweck wurden 88 krankheitsängstliche Patienten zufällig zwei Behandlungsbedingungen zugewiesen: Die Experimentalgruppe erhielt ein therapeutisch angeleitetes elektronisches TBT und die Kontrollgruppe nur die therapeutische Unterstützung, die wie in der TBT-Bedingung vier 50-minütige Einzelgespräche umfasste, jedoch keine weitere Intervention. Nach der zweiwöchigen Interventionsphase zeigte die TBT-Gruppe im Vergleich zur Kontrollgruppe weniger Krankheitsangst und beurteilte gesundheitsbedrohliche Stimuli als weniger erregend (Kerstner et al., 2015).

Auf diesen Befunden aufbauend beschäftigt sich die vorliegende Arbeit mit Fragen, die sich auf die KVT beziehen, die im Anschluss an ein TBT stattfand. Das TBT wurde der KVT zeitlich vorangestellt, weil wir an einer möglichst großen Stichprobe von Personen mit unterschiedlichen Schweregraden von Krankheitsangst interessiert waren und davon ausgingen, dass diese eher für eine Studienteilnahme zu gewinnen sind, wenn die für alle verbindliche Intervention kurz und neuartig ist.

\section{Fragestellung}

Wie häufig wird das optionale Angebot einer störungsspezifischen KVT von Patienten, die ihre Teilnahme für das kurze TBT zugesagt haben, in Anspruch genommen? Durch welche Personmerkmale kann die Inanspruchnahme vorhergesagt werden? Kann die Wirksamkeit der KVT durch das TBT noch gesteigert werden? Wie wirken sich TBT und KVT auf die Bewertung gesundheitsbedrohlicher Stimuli aus?

\section{Patienten und Methode}

Die 35 Patienten der vorliegenden Studie sind Teil einer größeren Stichprobe von krankheitsängstlichen Personen eines umfangreichen Forschungsprojekt zu verschiedenen Aspekten von Krankheitsangststörungen, das am Zentralinstitut für Seelische Gesundheit in Mannheim durchgeführt wurde (Bailer et al., 2013a,b, 2014). Die Teilnehmer wurden über unsere Ambulanzen für Psychiatrie, Psychosomatik und Psychologische Psychotherapie, über Anschreiben an niedergelassene Psychiater und Hausärzte sowie über Presseaufrufe, Flyer und Informationen auf der Homepage unseres Instituts rekrutiert. Das Studienprotokoll wurde von der lokalen Ethikkommission positiv begutachtet und die Studie im Einklang mit der Helsinki Deklaration durchgeführt.

Über ein zweistufiges Selektionsverfahren (siehe Abbildung 1) konnten 88 geeignete Personen gewonnen werden, die bereit waren, an einer zweiwöchigen Intervention mit jeweils vier therapeutischen Sitzungen teilzunehmen. Von diesen Personen erhielt die Hälfe zusätzlich ein elektronisches Tagebuchtraining (TBT), die andere Hälfte nicht. Drei Teilnehmer brachen aus Zeitgründen die Studie ab. Alle Teilnehmer mussten neben einem positiven Screeningbefund in mindestens einer von zwei Hypochondrieskalen (d.h. einem Summenwert $\geq 15$ im Short Health Anxiety Inventory (Salkovskis, Rimes, Warwick \& Clark, 2002) oder $\geq 8$ im WhiteleyIndex (Hiller \& Rief, 2004)) auch die Kriterien einer mindestens leichten Hypochondrie nach Fink und Kollegen (2004) erfüllen. Weitere Einschlusskriterien waren: Alter 18-65 Jahre, gute deutsche Sprachkenntnisse und das Vorliegen einer schriftlichen Einverständniserklärung. Ausschlusskriterien waren das Vorliegen einer Psychose, einer aktuellen Suchterkrankung sowie akute Suizidalität.

Im Anschluss konnten die Teilnehmer eine störungsspezifische KVT in Anspruch nehmen. Dieses freiwillige Angebot nutzten 38 Patienten, wobei drei Patienten die Behandlung schon nach wenigen Sitzungen (zwei aus Zeitgründen, einer wegen der Dominanz anderer psychischer Störungen) abbrachen. Bei Patienten mit komorbiden Achse-I-Störungen mussten die Krankheitsängste im Vordergrund stehen. 


\section{Interventionen}

Die Teilnehmer wurden randomisiert zwei Interventionsformen zugewiesen: Gruppe 1 erhielt vor der KVT ein elektronisches TBT. Die Teilnehmer wurden über einen Zeitraum von zwei Wochen trainiert, mit Hilfe eines elektronischen Tagebuchs mehrmals täglich zunächst ihre momentane Stimmung zu beurteilen, dann einen kurzen «mentalen Body-Scan» (Kabat-Zinn, 2005) durchzuführen, anschließend die Intensität von 12 kör-
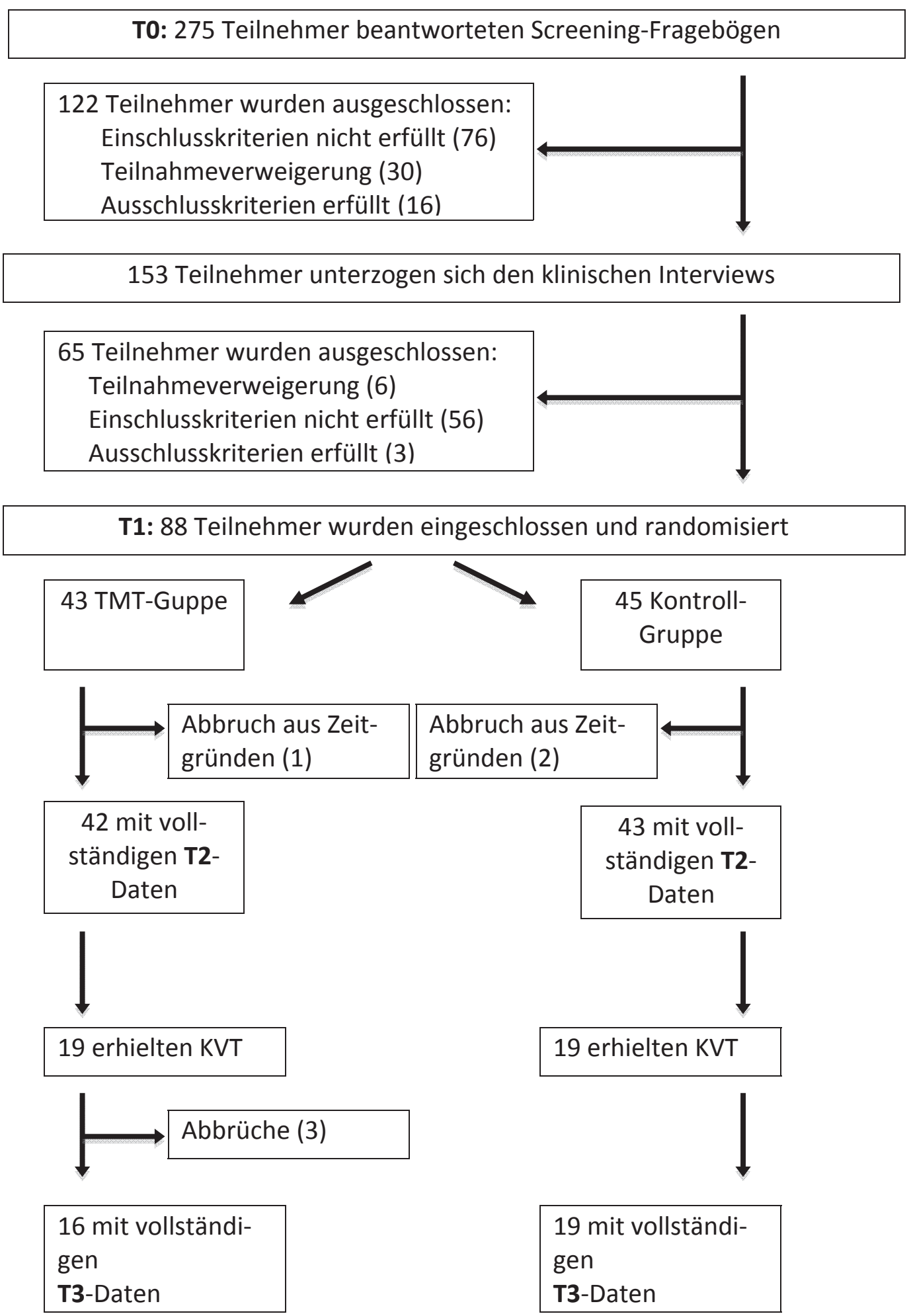

Abbildung 1. Flussdiagramm zum Studiendesign und Selektionsprozess der Teilnehmer. 
perlichen Beschwerden (Bauch-, Kopf-, Rücken-, Brustund Gelenkschmerzen, Schwindel, Ohnmachtsanfälle, Herzrasen, Kurzatmigkeit, Verstopfung, Übelkeit, sonstige Körperbeschwerden) zu beurteilen und abschließend neutrale oder psychologische Erklärungen für die körperlichen Beschwerden zu suchen. Die Körperbeschwerden stammen aus dem Gesundheitsfragebogen für Patienten (PHQ; Löwe, Spitzer, Zipfel \& Herzog, 2002). Schließlich wurde das Ausmaß der aktuellen Krankheitsangst eingeschätzt. Die gesamte Prozedur dauerte etwa drei Minuten. Die Teilnehmer wurden täglich zwischen 9 und 21 Uhr neun Mal zu festen Zeiten durch ein akustisches Signal ihres elektronischen Tagebuchs zur Durchführung dieser Prozedur aufgefordert. In den zwei Trainingswochen fanden zusätzlich vier 50-minütige Therapiesitzungen statt, um den «Body-Scan» einzuüben und die Teilnehmer beim TBT anzuleiten. Gruppe 2 ohne TBT erhielt vor der KVT ebenfalls vier 50-minütige Therapiesitzungen, die zur Erhebung der Biographie und der Krankheitsanamnese, der Analyse einer Krankheitsangstepisode sowie für Fragen zur Persönlichkeit genutzt wurden.

Die störungsspezifische KVT sah für jeden Patienten 16 Sitzungen manualisierte Einzelpsychotherapie vor, mit einer Frequenz von einer 50-minütigen Sitzung pro Woche. Das Therapiemanual orientierte sich eng an dem Manual von Bleichhardt und Weck (2010). Die acht psychologischen Therapeuten wurden durch die Autorin des Manuals (GB) geschult. Sie verfügten im Mittel über 4,7 Jahre (Range: 2-20 J.) VT-Erfahrung und führten alle audiovisuell aufgezeichnete Therapien unter regelmäßiger Supervision des Erstautors (JB) durch.

Die verbindlich vorgegebenen Inhalte der 16 Therapiesitzungen waren: Klärung der Behandlungsziele, Verhaltensanalyse zu einer Krankheitsangstepisode, kognitivbehaviorales Erklärungsmodell, Aufmerksamkeit und körperliche Symptome, Körperreise, Aufmerksamkeitsumlenkung, Auslöser von Krankheitsangst, alternative Bewertungen von Körpersymptomen, sokratischer Dialog und Disputation von Krankheitsängsten, Identifikation und Reduktion von sicherheitssuchendem Verhalten und Vermeidungsverhalten, Exposition an Körpersymptomen, Erstellung einer Hierarchie von Angstauslösern und Durchführung eines Habituationstrainings, Rückfallprophylaxe.

\section{Messinstrumente}

Alle Interessenten wurden zunächst hinsichtlich spezifischer psychopathologischer Merkmale gescreent $(\mathrm{T} 0=$ Screeningtermin) und erst bei einem Folgetermin $(\mathrm{T} 1=$ Diagnostiktermin) mit klinischen Interviews und weiteren Fragebögen untersucht. Die Wartezeit zwischen T0-T1 (Range: 1-34 Wochen) betrug für die Gruppe $1 \mathrm{im}$ Mittel $11(S D: 8)$ und für die Gruppe 2 etwa $12(S D: 8)$ Wochen $\left.t_{(86)}=0.68, p=.50\right)$.

\section{Selbstbeurteilungsskalen}

Der Whiteley-Index (Hiller \& Rief, 2004) und das Short Health Anxiety Inventory (SHAI; Bailer et al., 2013b; Salkovskis et al., 2002) wurden zur Erfassung von Krankheitsangst eingesetzt. Beide Skalen bestehen aus jeweils 14 Items. Sie besitzen gute psychometrische Eigenschaften und haben definierte Schwellenwerte für die Identifikation von hoch krankheitsängstlichen Personen. Zusätzlich wurden der Gesundheitsfragebogen für Patienten (PHQ; Kroenke, Spitzer, Williams \& Löwe, 2010; Löwe et al., 2002) zur Erfassung von 15 somatischen (PHQ-15) und 9 depressiven Symptomen (PHQ-9) genutzt. Außerdem wurden bei T1 Symptomattributionen mit dem Symptom Interpretation Questionnaire (SIQ; Glöckner-Rist, Prüfer, Rexroth \& Rist, 2012; Robbins \& Kirmayer, 1991) erhoben. Der SIQ, der sich in früheren Studien (Bailer, Rist, Witthöft, Paul \& Bayerl, 2005; Bailer, Witthöft, Bayerl $\&$ Rist, 2006) als reliabel und valide erwiesen hat, erfragt standardisiert Kausalattributionen für 13 häufige körperliche Beschwerden, die zu drei Subskalen zusammengefasst werden können: somatische, psychologische und normalisierende Attributionen.

Primäres Outcome-Kriterium war die Intensität der selbstberichteten Krankheitsangst, die über mehrere Messzeitpunkte $(\mathrm{T} 0=$ Screeningtermin, T1 = Diagnostiktermin/ Prä-TBT, T2 = Post-TBT und T3 = Post-KVT) hinweg mit dem mSHAI (Bailer et al., 2013) erfasst wurde. Der mSHAI unterscheidet sich vom SHAI nur durch ein modifiziertes Antwortformat, was allerdings zu einer großen Zeitersparnis bei der Bearbeitung führt. Der mSHAI ist somit eine ökonomische Alternative zur Originalskala und eignet sich insbesondere als Maß für die Verlaufskontrolle. Beide Skalen korrelieren sehr hoch miteinander $(r=.96$; Bailer et al., 2013b).

Zusätzlich wurden bei T1, T2 und T3 je zehn störungstypische Symptom- (Schwindel, Übelkeit, Kopfschmerzen, Durchfall, Atemnot, Schmerzen, Herzrasen, Erbrechen, Bauchschmerzen, Husten) und Krankheitswörter (Tumor, Bakterien, Viren, Hirnschlag, Herzinfarkt, Geschwür, Krebs, Infektion, Grippe, Ersticken) (Witthöft, Rist \& Bailer, 2008) mit Hilfe einer computergestützten Version des Self-Assessment Manikin (SAM) von Bradley und Lang (1994) auf den Dimensionen Valenz (9-stufige Antwortskala: «sehr angenehm - sehr unangenehm») und Arousal (9-stufige Antwortskala: «gar nicht aufregend sehr aufregend») beurteilt. Das SAM ist ein reliables nonverbales Verfahren zur affektiven Bewertung von Stimuli mit Hilfe von Piktogrammen.

\section{Klinische Interviews}

Alle aktuell vorhandenen psychischen Störungen wurden mit dem Strukturierten Klinischen Interview für DSM-IV Achse-I-Diagnosen (SKID-I) (Wittchen, Wunderlich, Gruschwitz \& Zaudig, 1997) erhoben. Im Anschluss wurde 
das Vorliegen einer hypochondrischen Störung nach der Definition von Fink et al. (2004) mit Hilfe eines von Fink und Kollegen entwickelten strukturierten klinischen Interviews überprüft. Die Interviews wurden von speziell geschulten und fortlaufend supervidierten Diagnostikern (6 Klinische Psychologinnen mit mehrjähriger SKID-Erfahrung) durchgeführt. In ersten eigenen Validierungsanalysen erwies sich das Fink-Interview als reliabel und valide (Bailer et al., 2013a).

\section{Statistische Verfahren}

Gruppenunterschiede wurden mittels $t$ - oder $\chi^{2}$-Tests auf Signifikanz getestet. Prädiktoren der Inanspruchnahme von KVT wurden mittels multipler logistischer Regressionsanalyse identifiziert. Die Auswirkungen der unterschiedlichen Behandlungen auf den Verlauf der Outcome-Kriterien wurden mit Hilfe von Varianzanalysen mit Messwiederholung untersucht. Berichtet werden die Greenhouse-Geisser korrigierten $F$ - und $p$-Werte für die Haupt- Interaktionseffekte. Als Maß der Effektstärke wird für die varianzanalytischen Ergebnisse das partielle eta ${ }^{2}$ berichtet (klein: $\eta_{p}{ }^{2}=.01$; mittel: $\eta_{p}{ }^{2}=.06$; groß: $\eta_{p}{ }^{2}=$ .14). Inter- und Intra-Gruppen-Effektstärken wurden mit Hilfe von Cohen's $d$ berechnet, wobei ein $d$ von 0.20 einem kleinen, ein $d$ von 0.50 einem mittleren und ein $d$ von 0.80 einem großen Effekt entspricht.

\section{Ergebnisse}

\section{Gruppenunterschiede}

Tabelle 1 enthält Angaben zu soziodemographischen und psychopathologischen Merkmalen der verschiedenen Subgruppen. Zunächst überprüften wir, ob sich die Teilnehmer, die nach der experimentellen Intervention eine KVT in Anspruch nahmen, von jenen, die das Angebot nicht nutzten, in den aufgelisteten Merkmalen unterschieden. Die Gruppenvergleiche erbrachten nur für zwei Merkmale signifikante Unterschiede: Patienten mit späterer KVT zeigten im SHAI und im Fink-HypochondrieInterview stärkere Krankheitsangst als jene ohne spätere KVT.

In einem weiteren Schritt überprüften wir mit einer schrittweisen multiplen logistischen Regressionsanalyse, ob die Inanspruchnahme der KVT durch die in Tabelle 1 aufgeführten Personmerkmale signifikant vorhergesagt werden kann. Dies war der Fall, wobei vier von 17 möglichen Prädiktoren ${ }^{1}$ in das Regressionsmodell aufgenommen wurden. Die Inanspruchnahme von KVT wurde durch höhere SHAI-Werte $(p=.018)$, höhere Schulbildung ( $p=$ $.021)$ und weibliches Geschlecht $(p=.041)$ signifikant vorhergesagt, zudem gab es einen Trend für den Prädiktor schwere Hypochondrie nach Fink $(p=.060)$. Die korrekte Klassifikationsrate betrug insgesamt 73,6\% (Modell $\chi^{2}=$ $21.71, p<.001$, Nagelkerke $\left.R^{2}=.296\right)$. Die Art der vorangegangenen Intervention (TBT versus kein TBT) hatte keinen Einfluss auf die Inanspruchnahme von $\operatorname{KVT}\left(\chi^{2}=\right.$ $0.08, p=.928$ ).

Im nächsten Schritt wurde überprüft, ob sich die beiden KVT-Gruppen in den aufgeführten Merkmalen signifikant voneinander unterschieden. Was mit einer einzigen Ausnahme - Einnahme von Antidepressiva - nicht der Fall war. Patienten ohne TBT nahmen signifikant häufiger Antidepressiva ein als jene mit TBT. Dieser Befund kann nicht durch signifikant höhere Depressionswerte bzw. eine signifikant höhere Rate an depressiven Störungen in dieser Gruppe erklärt werden².

\section{Verlauf der Krankheitsangst}

Eine Varianzanalyse mit Messwiederholung zur Prüfung von Mittelwertsunterschieden zwischen den beiden KVTGruppen in der mittels mSHAI zu T0, T1, T2 und T3 erfassten Krankheitsangst erbrachte sowohl einen signifikanten Zeiteffekt $\left(F_{(2.03,99)}=51.29, p<.001, \eta_{p}{ }^{2}=.61\right)$ als auch einen signifikanten Interaktionseffekt «Gruppe $x$ Zeit» $\left(F_{(2.03,99)}=3.29, p=.043, \eta_{p}^{2}=.09\right)$. Der ZwischenGruppen-Effekt war nicht signifikant $\left(F_{(1,33)}=3.73, p=\right.$ $\left..062, \eta_{p}^{2}=.102\right)$.

Die in Abbildung 2 dargestellten Symptomverläufe zeigen in der Gruppe mit TBT einen stärkeren Abfall der Krankheitsangst als in der Gruppe ohne TBT. Die Gruppen unterscheiden sich bereits zum Ende der zweiwöchigen experimentellen Interventionsphase $\mathrm{T} 2$ signifikant voneinander $\left(t_{(33)}=2.16, p=.039\right)$. Die errechnete InterGruppen-Effektstärke $d$ beträgt .75. Dieser Behandlungsvorteil zugunsten der Gruppe mit TBT war auch noch bei T3 (Post-KVT) in unveränderter Höhe $(d=.76)$ nachweisbar $\left(t_{(33)}=2.16, p=.039\right)$. Zusätzlich wurden für beide Behandlungsgruppen Prä(T1)-Post(T3)-Effektstärken ${ }^{3}$ bestimmt, diese lagen für beide Gruppen im hohen Bereich: (KVT mit TBT: $d=1.77$, KVT ohne TBT: $d=1.59$ ).

1 Prädiktoren waren: Alter, Schulbildung, Geschlecht, mSHAI, PHQ-9, PHQ-15, Fink-Hypochondrie, DSM-IV-Hypochondrie, irgendeine depressive Störung, irgendeine Angststörung, Anzahl DSM-Diagnosen, Antidepressiva, somatischer, psychologischer, normalisierender Attributionen, GAF-Wert, Interventionsart (ohne versus mit TBT).

2 Wir überprüften mit Kovarianzanalysen, ob die Einnahme von Antidepressiva signifikante Effekte auf die Outcome-Kriterien (mSHAI, SAM, Beschwerden und Stimmung) hatte, was nicht zutraf. Die Kovariate «Antidepressiva» erbrachte durchgängig nichtsignifikante Resultate $(F \mathrm{~s}<0.47 ; p \mathrm{~s}>.459)$ und hatte auch keinen signifikanten Einfluss auf die Haupt- und Interaktionseffekte. Diese Variable wurde daher in den nachfolgend dargestellten Varianzanalysen nicht kontrolliert.

3 Zur Berechnung der Intra-Gruppen-Effektstärke wurde die Prä-Post-Mittelwertsdifferenz an der gepoolten Prä-Post-Standardabweichung standardisiert. 
Tabelle 1

Stichprobenbeschreibung

\begin{tabular}{|c|c|c|c|c|c|c|}
\hline \multirow[t]{2}{*}{ Variable } & \multicolumn{3}{|c|}{ Experimentelle Intervention $(N=88)$} & \multicolumn{3}{|c|}{ KVT-Completer $(N=35)$} \\
\hline & $\begin{array}{l}\text { KVT: Nein } \\
(N=50)\end{array}$ & $\begin{array}{l}\text { KVT: Ja } \\
(N=38)\end{array}$ & $\begin{array}{l}t / \chi^{2}- \\
\text { value }\end{array}$ & $\begin{array}{l}\mathrm{KVT}_{\text {mit }} \mathrm{TBT}^{1} \\
\quad(N=16)\end{array}$ & $\begin{array}{l}\text { KVT ohne TBT } \\
\quad(N=19)\end{array}$ & $\begin{array}{l}t / \chi^{2}- \\
\text { value }\end{array}$ \\
\hline Alter $(M \pm S D)$ & $44.7 \pm 12.0$ & $41.9 \pm 11.2$ & $1.1 \mathrm{~ns}$ & $40.4 \pm 11.3$ & $44.5 \pm 11.4$ & $1.1 n s$ \\
\hline Geschlecht (\% weiblich) & 56.0 & 71.1 & $2.1 n s$ & 75.0 & 63.2 & $0.6 n s$ \\
\hline Schuljahre (\% $\geq 12 \mathrm{~J})$ & 58.0 & 71.1 & $1.6 n s$ & 75.0 & 78.9 & $0.1 n s$ \\
\hline Whiteley-Index $(M \pm S . D)$ & $10.2 \pm 2.0$ & $10.8 \pm 1.5$ & $1.6 n s$ & $10.7 \pm 1.7$ & $10.7 \pm 1.5$ & $0.9 n s$ \\
\hline Krankheitsangst (SHAI) $(M \pm S . D)$ & $27.4 \pm 4.6$ & $30.8 \pm 4.7$ & $3.3 * * *$ & $29.9 \pm 5.6$ & $30.6 \pm 3.7$ & $0.5 n s$ \\
\hline Depressive Symptome (PHQ-9) $(M \pm S . D)$ & $10.1 \pm 5.2$ & $10.9 \pm 5.2$ & $0.8 n s$ & $9.8 \pm 6.2$ & $11.1 \pm 4.1$ & $0.7 n s$ \\
\hline Somatische Symptome (PHQ-15) $(M \pm S . D)$ & $13.9 \pm 5.1$ & $14.4 \pm 5.0$ & $0.4 n s$ & $14.5 \pm 3.7$ & $13.0 \pm 5.1$ & $1.0 n s$ \\
\hline Somatische Symptomattributionen (SIQ-S) & $18.9 \pm 7.9$ & $20.1 \pm 8.2$ & $0.7 n s$ & $19.9 \pm 8.9$ & $18.3 \pm 6.3$ & $0.6 n s$ \\
\hline Psychologische Symptomattributionen (SIQ-P) & $23.0 \pm 8.0$ & $22.8 \pm 8.5$ & $0.8 n s$ & $22.5 \pm 9.0$ & $21.8 \pm 8.1$ & $0.3 n s$ \\
\hline Normalisierende Symptomattributionen (SIQ-N) & $17.6 \pm 6.4$ & $18.4 \pm 6.9$ & $0.6 n s$ & $19.1 \pm 7.0$ & $17.6 \pm 6.8$ & $0.7 n s$ \\
\hline $\begin{array}{r}\text { Krankheitsangststörung nach Fink: leicht }(\%) \\
\text { schwer }(\%)\end{array}$ & $\begin{array}{l}50.0 \\
50.0\end{array}$ & $\begin{array}{l}21.1 \\
78.9\end{array}$ & $7.7 * *$ & $\begin{array}{l}31.2 \\
68.8\end{array}$ & $\begin{array}{l}15.8 \\
84.2\end{array}$ & $1.2 n s$ \\
\hline \multicolumn{7}{|l|}{ Aktuelle DSM-IV Diagnose (\%): } \\
\hline Hypochondrie & 60.0 & 73.7 & $1.8 n s$ & 68.8 & 73.7 & $0.1 n s$ \\
\hline Somatisierungsstörung & 4.0 & 0 & $1.6 n s$ & 0 & 0 & $0.0 n s$ \\
\hline Schmerzstörung & 6.0 & 2.6 & $0.6 n s$ & 6.2 & 0 & $1.2 n s$ \\
\hline Majore Depression & 26.0 & 21.1 & $0.3 n s$ & 18.8 & 21.1 & $0.3 n s$ \\
\hline Dysthymia & 10.0 & 10.5 & $0.0 \mathrm{~ns}$ & 6.2 & 10.5 & $0.2 n s$ \\
\hline Generalisierte Angststörung & 8.0 & 13.2 & $0.6 n s$ & 18.8 & 10.5 & $0.5 n s$ \\
\hline Panikstörung & 38.0 & 39.5 & $0.0 n s$ & 43.8 & 31.6 & $0.6 n s$ \\
\hline Soziale Phobie & 12.0 & 15.8 & $0.3 n s$ & 12.5 & 10.5 & $0.0 n s$ \\
\hline Spezifische Phobie & 16.0 & 18.4 & $0.1 n s$ & 18.8 & 15.8 & $0.1 n s$ \\
\hline Zwangsstörung & 12.0 & 7.9 & $0.4 n s$ & 6.2 & 10.5 & $0.2 n s$ \\
\hline Posttraumatische Belastungsstörung & 4.0 & 2.6 & $0.1 n s$ & 0 & 0 & $0.0 n s$ \\
\hline Bulimia nervosa & 0 & 2.6 & $1.3 n s$ & 0 & 5.3 & $0.9 n s$ \\
\hline Anzahl aktueller DSM-IV Diagnosen $(M \pm S . D)$ & $2.0 \pm 1.7$ & $2.1 \pm 1.6$ & $0.4 n s$ & $2.1 \pm 1.6$ & $2.0 \pm 1.4$ & $0.2 n s$ \\
\hline Globales Funktionsniveau (GAF) $(M \pm S . D)$ & $66.5 \pm 11.5$ & $66.3 \pm 10.8$ & $0.1 n s$ & $66.9 \pm 10.1$ & $66.8 \pm 11.6$ & $0.0 n s$ \\
\hline Antidepressive Medikation & 34.0 & 26.3 & $0.6 n s$ & 6.2 & 42.1 & $5.9 *$ \\
\hline
\end{tabular}

Anmerkungen: ${ }^{1}$ Drei Abbrecher in der Anfangsphase der KVT, daher Reduktion des Ns von 19 auf 16 . TBT = elektronisches Tagebuchtraining. $* * * p<0,001, * * p<0,01, * p<0,05$

\section{Bewertung von gesundheitsbedrohlichen Stimuli}

Auch die über die Zeit (T1-T3) hinweg beobachtbaren Veränderungen in den affektiven Urteilen zu den gesundheitsbedrohlichen Stimuli (Symptom- und Krankheitswörter) wurden varianzanalytisch untersucht. Die Valenz- und Arousal-Urteile wurden jeweils getrennt für die beiden Wortkategorien (Symptome und Krankheiten) ausgewertet. Für jedes der vier Outcome-Kriterien konnte ein signifikanter Zeiteffekt nachgewiesen werden: (Valenz Symptome: $F_{(1.98,66)}=5.94, p=.004, \eta_{p}^{2}=.15$; Valenz Krankheiten: $F_{(1.67,66)}=6.80, p=.004, \eta_{p}^{2}=.17$; Arousal Symptome: $F_{(1.61,66)}^{(1.67,66)}=38.04, p<.001, \eta_{p}^{2}=.54$; Arousal Krankheiten: $\left.F_{(1.90,66)}=22.21, p<.001, \eta_{p}^{2}=.40\right)$. Die gesundheitsbedrohlichen Wortstimuli werden also mit fortschreitender Zeit als zunehmend weniger unangenehm und weniger erregend beurteilt.

Ferner fand sich ein signifikanter Interaktionseffekt «Gruppe $\times$ Zeit» für das Verlaufskriterium «Arousal-Symptome» $\left(F_{(1.61,66)}=5.22, p=.013, \eta_{p}^{2}=.14\right)$. Die Inspektion der in Abbildung 2 dargestellten Mittelwertsverläufe zeigt, dass bei der Gruppe mit TBT eine stärkere Habituation an die Symptomwörter stattfand als in der Kontrollgruppe. Der Gruppenunterschied ist bei T3 (Post-KVT) maximal ausgeprägt $(d=.42)$. Für die Arousal-Einschätzungen der Krankheitsbegriffe fand sich ein ähnliches Verlaufsmuster, der Interaktionseffekt erreichte jedoch nicht das konventionelle Signifikanzniveau $\left(F_{(1.90,66)}=2.67, p=.089, \eta_{p}{ }^{2}=\right.$ $.08)$. Die restlichen Interaktionseffekte $\left(F_{\mathrm{s}}<1.72, p \mathrm{~s}>\right.$ .11) und sämtliche Zwischen-Gruppen-Effekte $(F \mathrm{~s}<0.19$, ps > .67) waren nicht signifikant. 
A

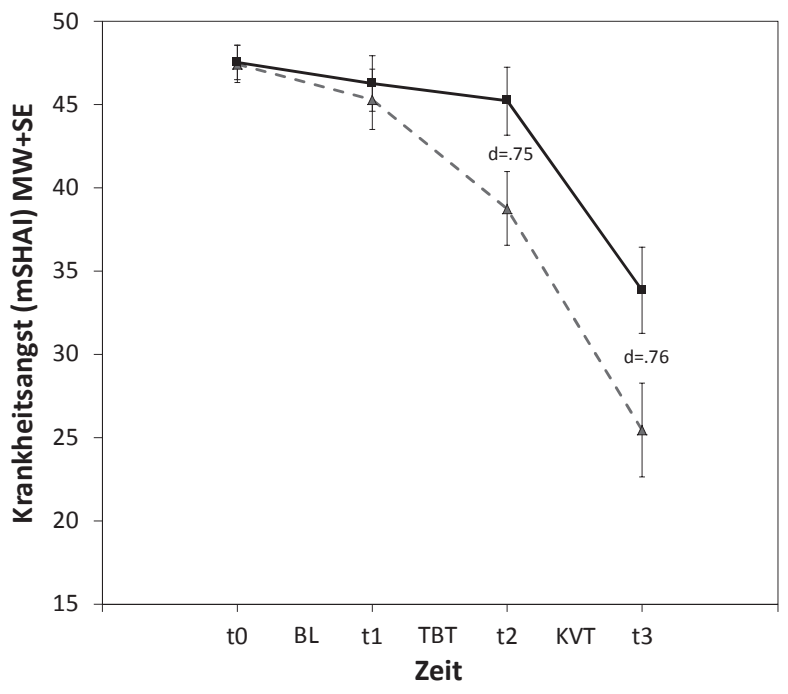

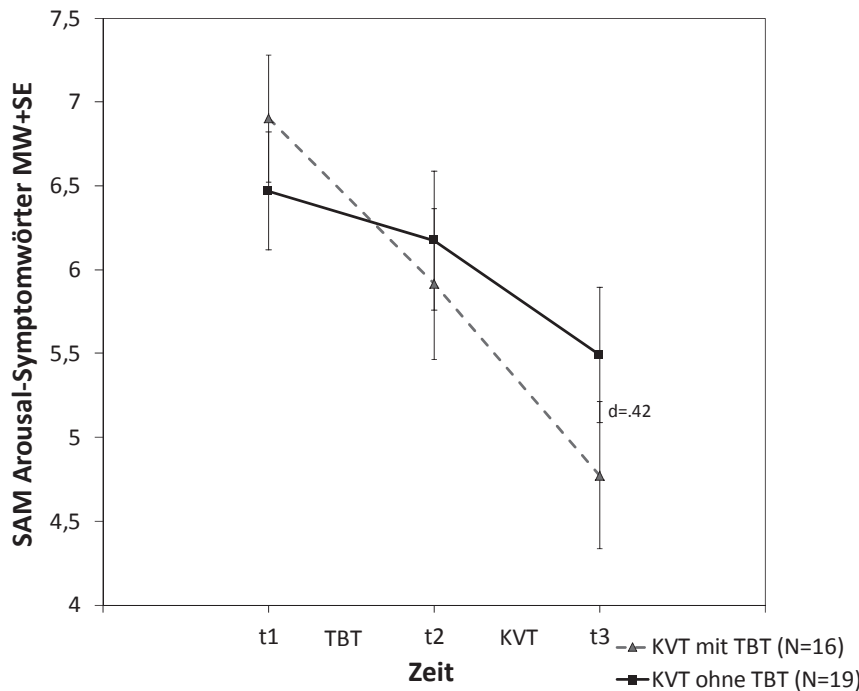

Abbildung 2. Verlauf der Krankheitsangst (A) und der Arousal-Ratings der Symptomwörter (B); BL = Baseline Datenerhebung, TBT = Elektronisches Tagebuchtraining, KVT = Kognitive Verhaltenstherapie; mSHAI = modified Short Health Anxiety Inventory, SAM = Self-Assessment-Manikin.

\section{Diskussion}

Die Resultate der vorliegenden randomisiert-kontrollierten Therapiestudie belegen erneut die Wirksamkeit von KVT bei Patienten mit Krankheitsangst. Wir konnten für diese Patientengruppe jedoch erstmals zeigen, dass sich die Effektivität der KVT durch den therapeutischen Einsatz von elektronischen Tagebüchern noch steigern lässt. Es wurden Handheld-PCs für die Durchführung eines zweiwöchigen TBTs zur Veränderung der Symptomattribution genutzt. Die Gruppe mit TBT zeigte im Vergleich zur Kontrollgruppe eine schnellere und stärkere Abnahme der Krankheitsangst. Die deutlichen Effekte des TBTs waren nicht nur unmittelbar nach der zweiwöchigen Interventionsphase nachweisbar $(d=.75)$, sondern in unveränderter Höhe auch noch vier Monate später, am Ende der störungsspezifischen KVT $(d=.76)$.

Weck und Kollegen (2013) erzielten in einer vergleichbaren Studie mit einem kurzen Aufmerksamkeitstraining, das allerdings nicht durch elektronische Assistenten unterstützt wurde, geringere Effekte $(d=.36)$. Für die unterschiedlich starken Effekte in diesen beiden Studien können verschiedene Faktoren verantwortlich sein. Die Studien unterscheiden sich sowohl hinsichtlich der gewählten Applikationsform (TBT versus therapeutisches Gespräch) als auch bezüglich der Trainingsinhalte (Modifikation von Aufmerksamkeit versus Symptomattribution). Zudem können Selektionseffekte eine Rolle spielen, zumal in der vorliegenden Studie nur die Daten jener 35 Patienten berücksichtigt wurden, die nach dem TBT auch noch eine KVT in Anspruch nahmen und diese nicht vorzeitig abgebrochen haben. Eine frühere Analyse aller Patienten $(N=85)$, die die experimentelle Interventionsphase (T1-T2) beendet hatten, lieferte dementsprechend einen etwas kleineren aber dennoch signifikanten Zwischen-Gruppen-Effekt ( $d=$ .62) zugunsten der TBT-Gruppe (Kerstner et al., 2015).

Prädiktoren für die Inanspruchnahme von KVT waren vor allem hohe Ausprägungen in der Krankheitsangst, aber auch weibliches Geschlecht und höhere Schulbildung. Wir schlussfolgern daraus, dass insbesondere Patienten mit hoher Krankheitsangst eher für eine relativ zeit- und arbeitsintensive KVT zu motivieren sind als Patienten mit leichteren Formen von Krankheitsangst. Letztere wären aber möglicherweise bereit, sich auf niederschwellige Therapieangebote wie z.B. Selbsthilfemanuale, internetbasierte KVT oder für den Handygebrauch programmierte KVT-Apps einzulassen.

Die Studie hat neben den bereits erwähnten Stärken auch einige methodische Schwächen: 1.) Aufgrund der geringen Fallzahl wurde auf Intention-to-Treat-Analysen verzichtet. 2.) Auch bei den gemessen an der Stichprobengröße komplexen Regressionsanalysen können wir ein «Over-Fitting» nicht gänzlich ausschließen. 3.) Eine a-priori Power-Analyse mit Bezug auf die geplanten Analysen wäre sinnvoll gewesen. 4.) Ferner liefert die Studie keine eindeutigen Hinweise auf den zentralen Wirkmechanismus, der dem TBT-Effekt zugrunde liegt. Wir können nur mit Bestimmtheit sagen, dass die durch das TBT initiierten Konfrontationen mit somatischen Beschwerden eine raschere und stärkere Veränderung in der Bewertung des Arousals von Wortstimuli sowie der Intensität der selbstberichteten Krankheitsangst zur Folge hatte. Ob diese Effekte jedoch durch Habituation, Reattribution oder eine Kombination aus beiden zustande gekommen sind, lässt sich aus den vorhandenen Ergebnissen nicht schließen. 5) Für die Abschätzung der klinischen Relevanz der Verbesserun- 
gen wäre eine Wiederholung des klinischen Interviews bei Therapieende wünschenswert gewesen. Wir müssen davon ausgehen, dass die Therapieeffekte im Interview insbesondere auf der Diagnoseebene (Störung noch vorhanden versus nicht vorhanden) geringer ausgefallen wären als in der hoch änderungssensitiven Symptomskala (mSHAI). Es bedarf daher weiterer Studien an größeren Stichproben, um die kurz- und langfristige Wirksamkeit der vorgestellten TBT zu belegen und die Frage nach dem Wirkmechanismus des TBTs zu klären.

Fazit: Die Wirksamkeit von KVT kann bei Patienten mit pathologischer Krankheitsangst durch ein zusätzliches zweiwöchiges elektronisches Tagebuchtraining noch gesteigert werden. Folglich werden elektronische Tagebücher und KVT-Apps in naher Zukunft wohl häufiger in der Einzel- und Gruppenpsychotherapie eingesetzt werden, da sie eine elegante, ökonomische und effektive Methode zur Verbesserung der Therapiewirksamkeit darstellen.

\section{Danksagung}

Die Studie wurde durch die Deutschen Forschungsgemeinschaft (DFG) unterstützt (BA 1597/5-1,2). Unser Dank gilt den nachfolgend aufgeführten Kolleginnen und Kollegen, die als Therapeuten und Diagnostiker bei der Datenerhebung mitgewirkt haben - Dr. Julia Ofer, Dr. Iris Wollgarten, Dr. Stefania Utzeri, Henriette Wagner, Dr. Kinga Muszer, Dr. Jana Strohmaier, Dr. Michael Gadinger und Dr. Silke Huffziger - sowie Prof. Dr. Ulrich Ebner-Priemer und Susanne Koudela für die technische Unterstützung bei der Programmierung der elektronischen Tagebücher.

\section{Literatur}

American Psychiatric Association (2000). Diagnostic and statistical manual of mental disorders (4th ed., text revision). Washington, DC: American Psychiatric Association.

American Psychiatric Association (2013). Diagnostic and statistical manual of mental disorders - DSM-5 (5th edition). Arlington: APA, American Psychiatric Association.

Bailer, J., Müller, T., Witthöft, M., Diener, C., Mier, D., Ofer, J. \& Rist, F. (2013a). Symptomattributionsstile bei Hypochondrie. Psychotherapeut, 57, 552-559.

Bailer, J., Rist, F., Müller, T., Mier, D., Diener, C., Ofer, J., ... Witthöft, M. (2013b). Erfassung von Krankheitsangst mit dem Short Health Anxiety Inventory (SHAI). Verhaltenstherapie \& Verhaltensmedizin, 34, 378-398.

Bailer, J., Rist, F., Witthöft, M., Paul, C. \& Bayerl, C. (2005). Evidence for overlap between idiopathic environmental intolerance and somatoform disorders. Psychosomatic Medicine, 67, 921-929.

Bailer, J., Witthöft, M., Bayerl, C. \& Rist, F. (2006). Syndrome stability and psychological predictors of symptom severity in idiopathic environmental intolerance (IEI) and somatoform disorders. Psychological Medicine, 37, 271-282.

Bailer, J., Witthöft, M., Wagner, H., Mier, D., Diener, C. \& Rist, F. (2014). Childhood maltreatment is associated with depres- sion but not with hypochondriasis in later life. Journal of Psychosomatic Research, 77, 104-108.

Barsky, A. J. \& Ahern, D. K. (2004). Cognitive behavior therapy for hypochondriasis: A randomized controlled trial. Journal of the American Medical Association, 291, 1464-1470.

Bleichhardt, G. \& Weck, F. (2010). Kognitive Verhaltenstherapie bei Hypochondrie. Heidelberg: Springer.

Bouman, T. K. (2014). Psychological treatments for hypochondriasis: a narrative review. Current Psychiaty Reviews, 10, 58-69.

Bradley, M. M. \& Lang, P. J. (1994). Measuring emotion: The self-assessment manikin and the semantic differential. Journal of Behaviour Therapy and Experimental Psychiatry, 25, 49-59.

Creed, F. \& Barsky, A. J. (2004). A systematic review of the epidemiology of somatisation disorder and hypochondriasis. Journal of Psychosomatic Research, 56, 391-408.

Eilenberg, T., Kronstrand, L., Fink, P. \& Frostholm, L. (2013). Acceptance and commitment group therapy for health anxiety - results from a pilot study. Journal of Anxiety Disorders, 27, 461-468.

Fink, P., Ornbol, E., Toft, T., Sparle, K. C., Forstholm, L. \& Olesen, F. (2004). A new, empirically established hypochondriasis diagnosis. American Journal of Psychiatry, 161, 1680-1691.

Glöckner-Rist, A., Prüfer, P., Rexroth, M. \& Rist, F., (2012), Deutscher Symptom Interpretation Questionnaire (SIQd). In A. Glöckner-Rist, A. (Hrsg.), Zusammenstellung sozialwissenschaftlicher Items und Skalen. ZIS Version 15.00. Bonn: GESIS.

Haby, M. M., Donnelly, M., Corry, J. \& Voss, T. (2006). Cognitive behavioural therapy for depression, panic disorder and generalized anxiety disorder: a meta-regression of factors that may predict outcome. Australian and New Zealand Journal of Psychiatry, 40, 9-19.

Hedman, E., Andersson, E., Andersson, G., Lindefors, N., Lekander, M., Rück, C. \& Ljótsson, B. (2013). Mediators in internet-based cognitive behavior therapy for severe health anxiety. PLoS One, 8, 77752.

Hedman, E., Andersson, G., Anderson, E., Ljótsson, B., Rück, C., Asmundson, G. J. G. \& Lindefors, N. (2011). Internetbased cognitive behavioural therapy for severe health anxiety: Randomised controlled trial. British Journal of Psychiatry, 198, 230-236.

Hedman, E., Lekander, M., Ljótsson, B., Lindefors, N., Rück, C., Hofmann, S. G., ... Schulz, S. M. (2014). Sudden gains in internet-based cognitive behaviour therapy for severe health anxiety. Behaviour Research and Therapy, 54, 22-29.

Hiller, W. \& Rief, W. (2004). German version of the WhiteleyIndex and the Illness Attitude Scales. Bern: Huber.

Hofmann, S. G. \& Smits J. A. (2008). Cognitive-behavioral therapy for adult anxiety disorders: a meta-analysis of randomized placebo-controlled trails. Journal of Clinical Psychiatry, 69, 621-632.

Kabat-Zinn, J. (2005). Gesund durch Meditation (11. Aufl.). Frankfurt: O. W. Barth.

Kerstner, T., Witthöft, M., Mier, D., Diener, C., Rist, F. \& Bailer, J. (2015). A diary-based modification of symptom attributions in pathological health anxiety: effects on symptom report and cognitive biases. Journal of Consulting and Clinical Psychology. http://dx.doi.org/10.1037/a0039056

Kroenke, K., Spitzer, R. L., Williams, J. B. W. \& Löwe, B. (2010). The Patient Health Questionnaire Somatic, Anxiety 
and Depressive Symptom Scales: a systematic review. General Hospital Psychiatry, 32, 345-359.

Löwe, B., Spitzer, R. L., Zipfel, S. \& Herzog, W. (2002). Gesundheitsfragebogen für Patienten (PHQ-D). Manual und Testunterlagen (2. Aufl.). Karlsruhe: Pfizer.

McManus, F., Surawy, C., Muse, K., Vazquez-Montes, M. \& Williams, J. M. G. (2012). A randomized clinical trial of mindfulnessbased cognitive therapy versus unrestricted services for health anxiety (hypochondriasis). Journal of Consulting and ClinicalPsychology, 80, 817-828.

Neng, J. M. \& Weck, F. (2013). Attribution of somatic symptoms in hypochondriasis. Clinical Psychology and Psychotherapy (in Druck). DOI:10.1002/cpp.1871.

Olatunji, B. O., Etzel, E. N., Tomarken, A. J., Ciesielski, B. G. \& Deacon B. (2011). The effects of safety behaviors on health anxiety: An experimental investigation. Behaviour Research and Therapy, 49, 719-728.

olde Hartman, T. C., Borghuis, M. S., Lucassen, P. L., van de Laar, F. A., Speckens, A. E. \& van Weel, C. (2009). Medically unexplained symptoms, somatisation disorder and hypochondriasis: Course and prognosis. A systematic review. Journal of Psychosomatic Research, 66, 363-377.

Rief, W., Hiller, W. \& Margraf, J. (1998). Cognitive aspects of hypochondriasis and the somatization syndrome. Journal of Abnormal Psychology, 107, 587-595.

Robbins, J. M. \& Kirmayer, L. J. (1991). Attributions of common somatic symptoms. Psychological Medicine, 21, 1029-1045.

Salkovskis, P. M., Rimes, K. A., Warwick, H. M. C. \& Clark, D. M. (2002). The Health Anxiety Inventory: Development and validation of scales for the measurement of health anxiety and hypochondriasis. Psychological Medicine, 32, 843-853.

Thomson, A. \& Page, L. (2007). Psychotherapies for hypochondriasis. Cochrane Database of Systematic Reviews, 4, CD006520.

Tyrer, P., Cooper, S., Salkovskis, P., Tyrer, H., Crawford, M., Byford, S., ... Barrett, B. (2014). Clinical and cost-effectiveness of cognitive behaviour therapy for health anxiety in medical patients: a multicentre randomised controlled trial. Lancet, 383, 219-225.

Warwick, H. M. \& Salkovskis, P. M. (1990). Hypochondriasis. Behaviour Research and Therapy, 28, 105-117.
Weck, F., Neng, J. M. B., Richtberg, S. \& Stangier, U. (2012a). The restrictive concept of good health in patients with hypochondriasis. Journal of Anxiety Disorders, 26, 792-798.

Weck, F., Neng, J. M. B., Richtberg, S. \& Stangier, U. (2012b). Dysfunctional beliefs about symptoms and illness in patients with hypochondriasis. Psychosomatics, 53, 148-154.

Weck, F., Neng, J. M. B. \& Stangier, U. (2013). The effects of attention training on the perception of bodily sensations in patients with hypochondriasis: A randomized controlled pilot trial. Cognitive Therapy and Research, 37, 514-520.

Wilhelm, P. \& Perrez, M. (2008). Ambulantes Assessment in der Klinischen Psychologie und Psychiatrie. Zeitschrift für Psychiatrie, Psychologie und Psychotherapie, 56, 169-179.

Williams, M. J., McManus, F., Muse, K. \& Williams, J. M. G. (2011). Mindfulness-based cognitive therapy for severe health anxiety (hypochondriasis): An interpretative phenomenological analysis of patients' experiences. British Journal of Clinical Psychology, 50, 379-397.

Williams, P. G. (2004). The psychopathology of self-assessed health: a cognitive approach to health anxiety and hypochondriasis. Cognitive Therapy and Research, 28, 629-644.

Wittchen, H. U., Wunderlich, U., Gruschwitz, S. \& Zaudig, M. (1997). Strukturiertes Klinisches Interview für DSM-IV Achse I: Psychische Störungen (SKID-I). Göttingen: Hogrefe.

Witthöft, M., Rist, F. \& Bailer, J. (2008). Enhanced early emotional intrusion effects and proportional habituation of threat response for symptom and illness words in college students with elevated health anxiety. Cognitive Therapy and Research, 32, 818-842.

World Health Organization (1993). The ICD-10 international statistical classification of mental and behavioral disorders: diagnostic criteria for research. Genf: WHO.

Prof. Dr. Josef Bailer

Zentralinstitut für Seelische Gesundheit

Abteilung Klinische Psychologie

68159 Mannheim

josef.bailer@zi-mannheim.de 